\title{
Hyperspectral Image Denoising with Composite Regularization Models
}

\author{
Ao Li, Deyun Chen, Kezheng Lin, and Guanglu Sun \\ Postdoctoral Research Station of Computer Science and Technology, Harbin University of Science and Technology, \\ Harbin 150080, China \\ Correspondence should be addressed to Ao Li; dargonboy@126.com
}

Received 21 October 2015; Revised 14 April 2016; Accepted 20 April 2016

Academic Editor: Pietro Siciliano

Copyright (C) 2016 Ao Li et al. This is an open access article distributed under the Creative Commons Attribution License, which permits unrestricted use, distribution, and reproduction in any medium, provided the original work is properly cited.

Denoising is a fundamental task in hyperspectral image (HSI) processing that can improve the performance of classification, unmixing, and other subsequent applications. In an HSI, there is a large amount of local and global redundancy in its spatial domain that can be used to preserve the details and texture. In addition, the correlation of the spectral domain is another valuable property that can be utilized to obtain good results. Therefore, in this paper, we proposed a novel HSI denoising scheme that exploits composite spatial-spectral information using a nonlocal technique (NLT). First, a specific way to extract patches is employed to mine the spatial-spectral knowledge effectively. Next, a framework with composite regularization models is used to implement the denoising. A number of HSI data sets are used in our evaluation experiments and the results demonstrate that the proposed algorithm outperforms other state-of-the-art HSI denoising methods.

\section{Introduction}

Hyperspectral image (HSI) processing is one of the most powerful techniques in remote sensing. It has a wide range of applications in mineral exploration [1], environmental monitoring [2-4], military surveillance $[5,6]$, and other related fields. Despite the development of advanced techniques in hyperspectral sensors, noise is unavoidably introduced in the acquisition process, which limits HSI applications and has a negative impact on many subsequent tasks, such as unmixing, classification, and object detection. Therefore, HSI denoising is a classic problem that is still an active area of research in HSI preprocessing.

In the past decades, many algorithms have been proposed for noise reduction in HSI. A simple idea is to adopt a conventional denoising method for natural images bandby-band or pixel-by-pixel. However, these methods cannot obtain satisfactory results because they ignore the spectral correlation between different bands, which is of great importance for improving denoising quality. At the same time, the spectral correlation is destroyed by the above methods and produces extra distortion. Hence, the spatial and spectral dimensions should be considered simultaneously. Othman and Qian proposed a noise reduction scheme using a wavelet shrinkage based hybrid spatial-spectral derivative domain in which the noise level is elevated. The method utilized the dissimilarity of signal regularity in the corresponding spatial and spectral dimensions [7]. Assuming that the image noise exists in the low-energy principal component analysis (PCA) channels, Chen and Qian proposed a denoising algorithm based on PCA and wavelets [8]. In their algorithm, 2D bivariate wavelet thresholding is used to remove the noise in lowenergy PCA channels and a 1D dual-tree complex wavelet transform (DCWT) based denoising method is utilized to remove the spectrum noise of the HSI data cube.

Inspired by the excellent performance of the total variation method for natural image denoising, in [9-11], a cubic total variation model was proposed for HSI denoising that combined the $2 \mathrm{D}$ total variation in the spatial domain with the $1 \mathrm{D}$ total variation in the spectral dimension and achieved competitive image quality [12]. Yuan et al. then proposed an adaptive spectral-spatial total variation model in which the differences of spectral noise and spatial information are both considered to better suppress the noise [13]. Motivated by this method, Liao et al. proposed a two-stage method that combines kernel principal component analysis (KPCA) and 
total variation, which obtained promising denoising results [14].

Recently, sparse representation (SR) based methods have been attracting more attention in image denoising as a powerful prior model [15-18]. In the SR framework, the signal is assumed to be represented by a liner combination of a few atoms in a learned or designed dictionary. Based on this viewpoint, the true signal can be recovered from its noisy observation with an ill-posed inverse problem by enforcing the sparsity prior. Rasti et al. proposed a new liner model that modeled the HSI with singular value decomposition and wavelets and then reconstructed the true image based on sparse regularization [19]. This approach achieves some visual improvement and can be applied to large data sets. In [20], a sparse low rank model for HSI was used to solve the denoising problem. The two benefits of the proposed model are dimensionality reduction with PCA and the exploitation of DCWT coefficients associated with the principle components separately. To better utilize the local and global redundancy in the spatial and spectral domains of HSI, Zhao and Yang [21] proposed a method using SR and the low rank constraint, which can be thought as utilizing two regularizers to constrain the local and global redundancy respectively and shows state-of-the-art performance.

In light of these methods, in this paper, we propose a novel algorithm for HSI denoising using composite regularization models. First, based on the patch SR, we present a specific way to extract the patches from the HSI by considering the information in both the spatial and spectral dimensions. On the one hand, this approach could enable the extracted patches to maintain their correlation in the local spectral dimension. On the other hand, with a nonlocal technique (NLT), it could also exploit the nonlocal self-similarity to preserve the detailed texture information in the spatial domain. In view of the excellent performance of the prior constraint for denoising, a framework with two composite regularization models (total variation and nonlocal group sparsity) is proposed for HSI denoising. Furthermore, to solve the nonconvex objective function in the framework, the alternating direction method of multipliers (ADMM) is utilized to obtain the approximate solution to the inverse problem.

The rest of the paper is organized as follows. In Section 2, basic theory for image denoising based on regularization will be introduced. Section 3 presents the proposed framework with composite regularization models for HSI denoising and its numerical solution method. The comparison experiments and results are presented in Section 4 . Section 5 concludes the paper.

\section{Regularization Model Based Image Denoising}

Image denoising aims to recover the true image from its noisy observation, which can be formulated as

$$
f=u+v
$$

where $f \in R^{N}$ is the degradation observation, $N$ is the whole pixel numbers in image, $u \in R^{N}$ is the true image, and $v \sim N(0, \sigma)$ is the zero-mean additive Gaussian noise. To get the estimation image $\widehat{u}$ as close as possible to the noise-free image $u$, a solution with $L_{2}$-norm constraint can be obtained as

$$
\widehat{u}=\underset{u}{\arg \min }\left\{\|f-u\|_{2}^{2}\right\}
$$

where $\|\cdot\|_{2}$ denotes the $L_{2}$-norm operator and $\|f-u\|_{2}$ is called data fidelity term. Due to the nonunique solution for the inverse problem in (2), the prior knowledge is utilized to supply a better solution space by regularizing the above illposed problem. So the formulation in (2) can be rewritten as follows:

$$
\widehat{u}=\underset{u}{\arg \min }\left\{\|f-u\|_{2}^{2}+\eta \varphi(u)\right\},
$$

where $\varphi(u)$ is the prior constraint on the true image, which will help to obtain a better solution and improve the image quality. $\eta$ is a scalar parameter to balance the above two terms.

It is widely known that prior knowledge plays an essential role in the regularization of a denoising algorithm. From the formulation in (3), we can also see that different priors produce different performance. Hence, designing a term to effectively reflect the prior is a critical issue in image denoising. Total variation is a classical regularization model that is widely used in image restoration, in which it is assumed that the image is locally smooth except at its discontinuous edges. Therefore, it could oversmooth the texture and achieve worse performance in fine structures, as it only accounts for the local statistical characteristics. Some additional studies on total variation have been proposed to further improve its denoising quality $[22,23]$.

Another popular model that has led to promising result for image denoising is patch-based sparsity prior knowledge. The sparse model assumes that each patch from an image can be represented by a few atoms of a dictionary. Thanks to a number of coding methods, the noise-free patches can also be accurately recovered using effective $l_{0}$-norm minimization or its relaxed version, $l_{1}$-norm minimization. Typical methods to do this include the basis pursuit [24], matching pursuit [25], iterative thresholding [26], and split Bregman [27] methods.

In recent years, the NLT has attracted a large amount of attention for its powerful performance on sharper edges and ability to preserve fine-scale details. Because of the large amount of self-similarity exhibited in natural images from pixel to block level, a number of NLT-based regularization terms have been exploited for image inverse problem [28-30].

Although the regularization model successfully denoises images, there remain some shortcomings when it is used in HSI denoising. The first is that the aforementioned conventional regularization models can only be used band-byband in HSI denoising. This ignores the significant correlation between spectral dimensions and produces additional spectral distortion. The second is that the single prior model used for denoising is not enough to obtain superior results. Hence, in the next section, we discuss how to solve these two shortcomings using our proposed novel framework. 


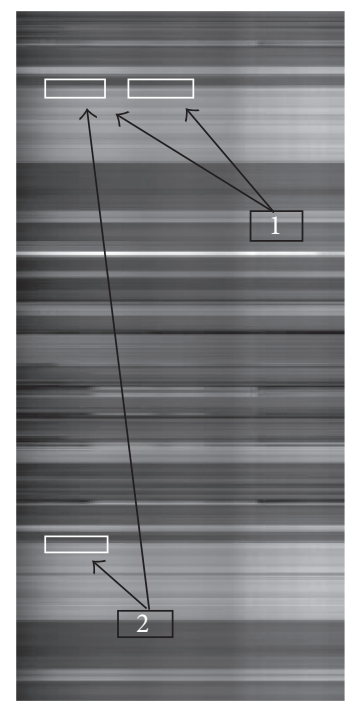

Figure 1: The reshaped 2D matrix for San Diego.

\section{Proposed Composite Regularization Model Framework}

3.1. Patch-Based Extraction Method. For HSI denoising, to obtain better recovery quality, the patch extracting way should be designed carefully. To consider the spatial and spectral dimension simultaneously, a simplest way is to extract local cube patches from HSI directly. However, the problem of this way is that there are too many pixels in each cube with only a small size, such as $8 \times 8 \times 3$, which will cost huge computational complexity for solving the regularization optimization. Meanwhile, incorporating the NLT, some extra cost will be produced due to the cube matching. Furthermore, large error will be caused when the Euclidean distance between cubes is measured with increased dimension.

Hence, an effective means to extract the patch is key to HSI denoising. To avoid the above problem, we propose a specific way to extract the patch by considering the spatialspectral information adaptively. To this end, we vectorize the images in each band from the HSI and then rearrange them as the columns of a new $2 \mathrm{D}$ matrix $U$, which can be expressed as

$$
U=\left[\begin{array}{llll}
u_{1} & u_{2} & \cdots & u_{l}
\end{array}\right]
$$

where $u_{p} \in R^{M N} \quad(p=1, \ldots, l)$ is the vectorized image in each band, $M$ and $N$ are the number of rows and columns of the band image, and $l$ is the number of spectral bands.

We take the image San Diego as an example. We reshape it into a $2 \mathrm{D}$ matrix, part of which is shown in Figure 1. As illustrated in Figure 1, the rows and columns of this 2D matrix present the spatial and spectral dimensions of the original band image separately. That is, we transform the spatialspectral information included in the HSI cube into a more compact $2 \mathrm{D}$ form.

The advantages of extracting patches from the reshaped 2D matrix are as follows. As shown in Figure 1, the patch with a white border includes the pixels located in the same spatial position within neighboring spectral bands. This is utilized as a unit in our framework, which employs the spatial-spectral information jointly. In Figure 1, the two similar patches in group 1 are spectrally correlated with each other because of their neighboring locations in the spectral dimension of the reshaped 2D matrix. Nevertheless, other similar patches in group 2 come from the same neighboring spectral bands with different spatial location. This indicates that the specific patch extraction method supplies a new way to exploit the spatial and spectral information simultaneously. That means we can construct a certain patch-based prior to constrain the nonlocal self-similarity across the spectral and spatial information by easily extracting patch from reshaped $2 \mathrm{D}$ images just as the general patch extracting method in [28], which will lead to a better performance on preserving fine-scale structures inevitability for HSI denoising problem.

3.2. Optimization Problem in the Proposed Framework. To the best of our knowledge, the single prior cannot be enough to obtain promising results. For example, to improve the conventional single total variation model, Liu et al. proposed integrating the nonlocal prior into total variation to take advantage of both of them, which achieved better reconstruction performance for CT image [31]. Similarly, Dong et al. exploited the nonlocal self-similarity in natural images and proposed a nonlocal central coding to impose the image to approximate to its clustering center under sparse representation [32]. Therefore, inspired by the achievement of total variation and NLT-based group sparsity, we propose a creative HSI denoising framework with them jointly, which can be expressed as

$$
\min _{u}\left\{\frac{1}{2}\|f-u\|_{2}^{2}+\eta \mathrm{TV}(u)+\tau \operatorname{NGSR}(u)\right\},
$$

where $f$ and $u$ denote the vectoring reshaped observation 2D matrix and the true HSI $2 \mathrm{D}$ matrix. $\tau$ and $\eta$ are the positive scalar parameter to control the balance between the above three terms.

The second term $\mathrm{TV}(u)$ presents the total variation prior model, which can be formulated as

$$
\operatorname{TV}(u)=\|D u\|_{1},
$$

where $D=\left[D_{v}, D_{h}\right]$ is the synthesis gradient operator and $D_{v}$ and $D_{h}$ are the vertical and horizontal difference operator, which is well studied in many literatures $[31,32]$.

The third term NGSR $(u)$ presents the NLT-based group sparse representation prior model denoted by

$$
\mathrm{NGSR}=\sum_{i, j}\left\|W^{T} G\left(u_{i, j}\right)\right\|_{p, q},
$$

where $u_{i, j}$ denotes a patch centered on the element of reshaped $2 \mathrm{D}$ matrix located in $(i, j) . G\left(u_{i, j}\right)=\left[u_{i, j}, u_{i, j}^{1}, u_{i, j}^{2}\right.$, $\left.\ldots, u_{i, j}^{k}, \ldots\right]$ presents a matrix that takes the similar patches to $u_{i, j}$ as its columns, and $k$ is the index number. $W$ is the wavelet dictionary, which is proved to be an effective tool to represent the image. $\|A\|_{p, q}$ is norm regularization to constrain the 
group sparsity of matrix $A$, whose definition can be denoted by

$$
\|A\|_{p, q}=\sum_{i}\left\|\alpha^{i}\right\|_{p}^{q}
$$

where $\alpha^{i}$ denotes the $i$ th row of matrix $A$.

In the optimization in (5), the second term constrains and guarantees the local smoothness, and the third term preserves finer structures by constraining the group sparsity with self-similarity in nonlocal patches. Moreover, because of the specific way patches are extracted, the framework not only utilizes the composite prior constraints, but also exploits the spatial-spectral information jointly. It is believed that better denoising results will be achieved by our proposed framework.

\section{Numerical Strategy for Solving the Optimization}

In this section, we present the details of the numerical strategy to solve the minimization problem in the proposed framework. The objective function in (5) can be solved for its composite prior constraints via a variable splitting technique. ADMM is a popular variable splitting technique for solving complex minimization that is also widely used in many image inverse problems [33].

Consider the following constrain optimization:

$$
\begin{array}{ll}
\min & \{Q(u)+g(\omega)\} \\
\text { s.t. } & A u+B \omega=C,
\end{array}
$$

where $Q(\cdot)$ and $g(\cdot)$ are two functions with variable $u$ and $\omega$.

To solve the minimization in (9), we can resort to its augmented Lagrangian function with unconstraint form:

$$
\begin{aligned}
L_{\rho}(u, \omega, p)= & Q(u)+g(\omega)+p^{T}(A u+B \omega-C) \\
& +\frac{\rho}{2}\|A u+B \omega-C\|_{2}^{2} .
\end{aligned}
$$

And then, minimize the equation in (10) based on ADMM with the following three optimization subproblems to obtain the solution iteratively:

$$
\begin{aligned}
& u^{k+1}=\underset{u}{\arg \min }\left\{Q(u)+\frac{\rho}{2}\left\|A u+B \omega^{k}-C+p^{k}\right\|_{2}^{2}\right\}, \\
& \omega^{k+1} \\
& \quad=\underset{\omega}{\arg \min }\left\{g(\omega)+\frac{\rho}{2}\left\|A u^{k+1}+B \omega-C+p^{k}\right\|_{2}^{2}\right\}, \\
& p^{k+1}=p^{k}+\left(A u^{k+1}+B \omega^{k+1}-C\right) .
\end{aligned}
$$

We return to the minimization problem in (5) and present the implementation details of our improved ADMM. First, we impose $A=\left[\begin{array}{l}I^{N \times N} \\ I^{N \times N}\end{array}\right], B=-I^{2 N \times N}$, and $C=\mathbf{O}$, where $I$ and $\mathbf{O}$ denote the identity matrix and null matrix, respectively. And then, we define $\omega=\left[\begin{array}{c}z \\ v\end{array}\right]$ and obtain

$$
A u+B \omega=\left[\begin{array}{l}
I \\
I
\end{array}\right] u-\left[\begin{array}{l}
z \\
v
\end{array}\right]=\mathbf{O}
$$

To adapt the minimization (5), we also impose the two following functions:

$$
\begin{aligned}
& Q(u)=\frac{1}{2}\|f-u\|_{2}^{2}, \\
& g(\omega)=\eta \operatorname{TV}(z)+\tau \operatorname{NGSR}(v) .
\end{aligned}
$$

So the objective function in (5) can be rewritten as

$$
\begin{array}{ll}
\min _{u, z, v} & \left\{\frac{1}{2}\|f-u\|_{2}^{2}+\eta \operatorname{TV}(z)+\tau \operatorname{NGSR}(v)\right\} \\
\text { s.t. } & {\left[\begin{array}{l}
z \\
v
\end{array}\right]=\left[\begin{array}{l}
u \\
u
\end{array}\right] .}
\end{array}
$$

In terms of (11)-(13), we impose auxiliary variable $p^{k}$ in (13) to be $p^{k}=\left[\begin{array}{c}q^{k} \\ t^{k}\end{array}\right]$ and obtain their extension forms as follows:

$$
\begin{gathered}
u^{k+1}=\underset{u}{\arg \min }\left\{\frac{1}{2}\|f-u\|_{2}^{2}\right. \\
\left.+\frac{\rho}{2}\left\|\left[\begin{array}{l}
u \\
u
\end{array}\right]-\left[\begin{array}{l}
z^{k} \\
v^{k}
\end{array}\right]+\left[\begin{array}{c}
q^{k} \\
t^{k}
\end{array}\right]\right\|_{2}^{2}\right\} \\
{\left[\begin{array}{l}
z^{k+1} \\
v^{k+1}
\end{array}\right]=\underset{z, v}{\arg \min }\{\eta \operatorname{TV}(z)+\tau \operatorname{NGSR}(v)} \\
\left.+\frac{\rho}{2}\left\|\left[\begin{array}{l}
u^{k+1} \\
u^{k+1}
\end{array}\right]-\left[\begin{array}{l}
z \\
v
\end{array}\right]+\left[\begin{array}{l}
q^{k} \\
t^{k}
\end{array}\right]\right\|_{2}^{2}\right\} \\
{\left[\begin{array}{l}
q^{k+1} \\
t^{k+1}
\end{array}\right]=\left[\begin{array}{l}
q^{k} \\
t^{k}
\end{array}\right]+\left[\begin{array}{l}
u^{k+1} \\
u^{k+1}
\end{array}\right]-\left[\begin{array}{l}
z^{k+1} \\
v^{k+1}
\end{array}\right] .}
\end{gathered}
$$

For more compact expression, the above subproblems are further separated as

$$
\begin{aligned}
u^{k+1} & =\underset{u}{\arg \min }\left\{\frac{1}{2}\|f-u\|_{2}^{2}+\frac{\rho}{2}\left\|u-z^{k}+q^{k}\right\|_{2}^{2}\right. \\
+ & \left.\frac{\rho}{2}\left\|u-v^{k}+t^{k}\right\|_{2}^{2}\right\}, \\
z^{k+1} & =\underset{z}{\arg \min }\left\{\frac{\rho}{2}\left\|z-u^{k+1}-q^{k}\right\|_{2}^{2}+\eta \operatorname{TV}(z)\right\}, \\
v^{k+1} & =\underset{z}{\arg \min }\left\{\frac{\rho}{2}\left\|v-u^{k+1}-t^{k}\right\|_{2}^{2}+\tau \operatorname{NGSR}(v)\right\} .
\end{aligned}
$$


The extension auxiliary variables $p^{k}$ can also be updated as

$$
\begin{aligned}
& q^{k+1}=q^{k}+u^{k+1}-z^{k+1} \\
& t^{k+1}=t^{k}+u^{k+1}-v^{k+1}
\end{aligned}
$$

Since the minimization in (18) is a quadratic function, a closed-form solution can be obtained as

$$
u^{k+1}=\frac{1}{1+2 \rho}\left(f+\rho\left(z^{k}-p^{k}\right)+\rho\left(v^{k}-t^{k}\right)\right) .
$$

The subproblem in (19) can be regarded as a standard total variation problem. Many equally good algorithms have been proposed to solve total variation such as fast iterative shrinkage threshold algorithm (FISTA) [34] and the split Bregman-based method [35]. In our paper, we adopted FISTA to guarantee fast convergence.

The third minimization in (20) is a joint sparsity coding problem, and the group coding can be computed by the method in [17]. Thus, $v^{k+1}$ is obtained by averaging the products of the wavelet dictionary and group coding.

The specific steps of the overall method are described. follows:

Detailed numerical strategy for proposed framework is as

Input. The noisy observation HSI $f$;

Initialization. $u^{0}=f, z^{0}=v^{0}=\mathbf{0}, p^{0}=t^{0}=\mathbf{0}, k=0$;

Steps

(1) Update $u^{k+1}$ with formulation (22);

(2) Compute $\gamma=\eta / \rho$;

(3) Update $z^{k+1}=\operatorname{argmin}_{z}\left\{(1 / 2)\left\|z-u^{k+1}-q^{k}\right\|_{2}^{2}+\gamma\|D z\|_{1}\right\}$ with FISTA;

(4) Compute $\lambda=\tau / \rho$;

(5) Update $v^{k+1}=\operatorname{argmin}_{z}\left\{(1 / 2)\left\|v-u^{k+1}-t^{k}\right\|_{2}^{2}+\right.$ $\left.\lambda \sum_{i, j}\left\|W^{T} G\left(v_{i, j}\right)\right\|_{p, q}\right\}$ with the method in [17];

(6) Updated the auxiliary variables with formulation (21);

$$
k \longleftarrow k+1
$$

Until criterion satisfied;

Experimental Results and Analysis. To verify the performance of our proposed framework, we applied it to two different HSI data sets, San Diego and Indian Pines. To evaluate it with respect to other methods, we compared the proposed method with the cubic total variation model (CTVM) [12], two-stage denoising method (TwoS) for HSI [14], and PCA based HSI denoising using wavelet shrinkage (PCA+WS) [8]. The assessment indicators are the peak signal-to-noise ratio (PSNR) and recently popular structural similarity (SSIM), which is based on the human vision system [36]. To evaluate the synthesis denoising performance, we plot the mean values of PSNR and SSIM of all the test bands in the HSI data. Considering that some bands of the experimental data sets
TABLE 1: The overall accuracy of classification with several methods.

\begin{tabular}{cccccc}
\hline & Noisy & PCA+WS & CTVM & TwoS & Ours \\
\hline OA & $75.8 \%$ & $83.2 \%$ & $84.3 \%$ & $86.0 \%$ & $87.5 \%$ \\
\hline
\end{tabular}

are heavily corrupted by noise and hence cannot be used as ground truth, we visually selected some cleaner continuous bands as the ground truth test data for the simulation. In the following experiments, the parameters in our proposed algorithm were set only according to the noise level, independently of the test data. The parameters for other comparison methods were set to the original values in their papers. The window size for similar patch search was set to $20 \times 20$ and the patch size was $8 \times 8$. In our experiment, we fixed the number of similar patches in every group, which enabled (20) to be solved more efficiently. The standard deviation $\sigma$ of the noise was varied from 5 to 20 in increments of 5 . Before the experiment, the extracted testing data were normalized to $[0,1]$. Specifically, the atmospheric and water absorption bands were removed from Indian Pines before the test data was extracted.

Because of space limitations, we only present the denoising results of one band (the 40th band) of San Diego using different methods and different standard deviations of the noise. These results are shown in Figures 2 and 3.

It is clear from the visual comparison in Figures 2 and 3 that PCA+WS obtains the worst visual performance among these methods because it accounts for the spatial and spectral dimensions separately, which eradicates many details found in the ground truth. Considering both the difference in the spectral noise and spatial information, CTVM retains more edges compared to PCA+WS because of its spatial-spectral technique. However, it strongly enforces local smoothness, which produces some mottled blocks in the denoised results. In contrast to PCA+WS, TwoS uses kernel PCA to first reduce the uncorrelated noise and then separate out the most significant information. Then, it removes the remaining noise from the low-energy channels with fast primal-dual total variation. Hence, the visual results of TwoS look better with sharper textures than those of PCA+WS. For example, TwoS shows more details of the parking apron at the bottom left, which is often used as a target in HSI target detection. Compared to the other algorithms, our method shows an even more promising performance. It not only suppresses most of the noise, but also preserves more details and sharp edges. Furthermore, from the plots of the mean PSNR and SSIM given in Figure 4, we conclude that our method outperforms other comparative algorithms with respect to these metrics.

To validate the further effectiveness of our proposed method, classification experiments for HSI are tested on India Pines. 10 percent of the numbers of testing samples are employed as the training samples. We take the support vector machine as the classifier and categorizes the image into 16 classes. The classification results are depicted in Figure 5. It is clear that the accuracy is improved greatly after denoising processing. Also, to more obviously, we list the overall accuracy $(\mathrm{OA})$ in Table 1 . The highest $\mathrm{OA}$ of proposed method 


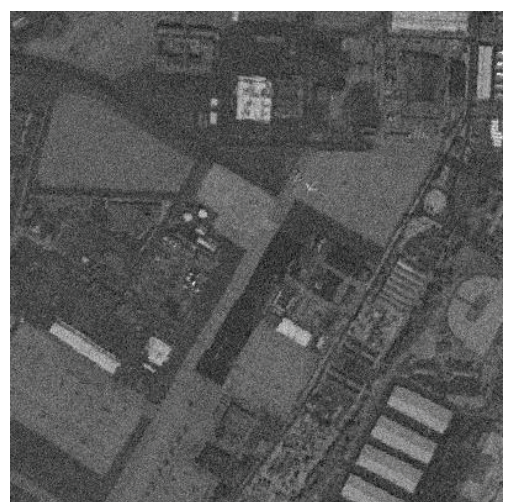

(a) Noisy HSI

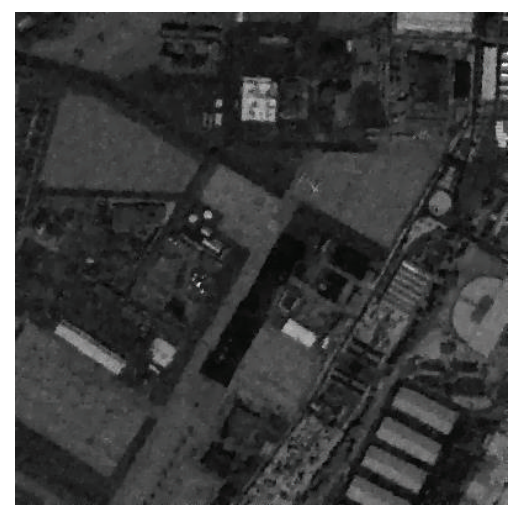

(b) CTVM

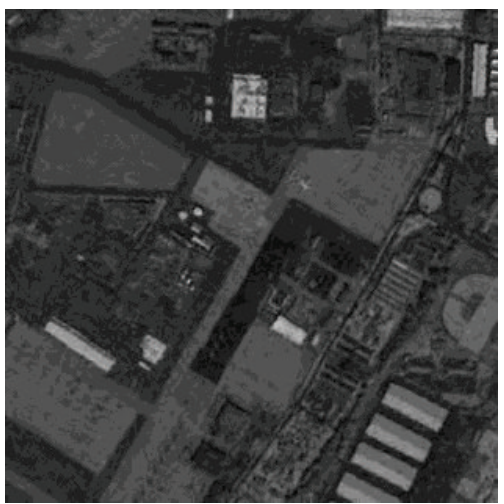

(c) TwoS

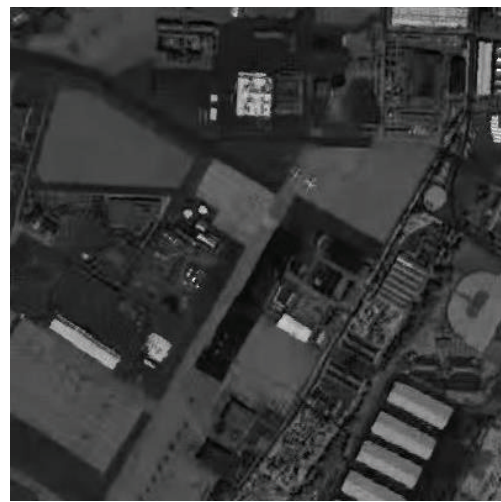

(d) PCA+WS

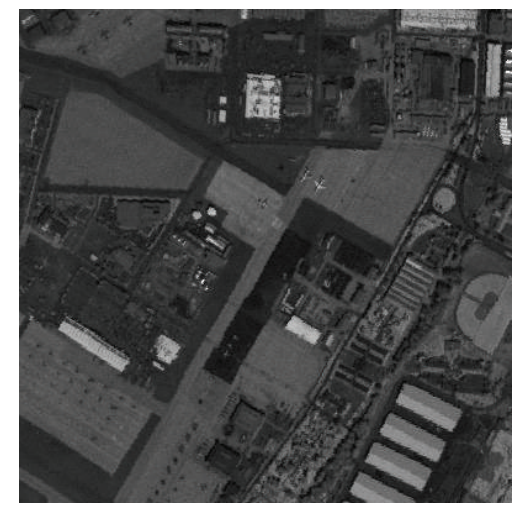

(e) Our method

FIGURE 2: Denoising results with $\sigma=20$.

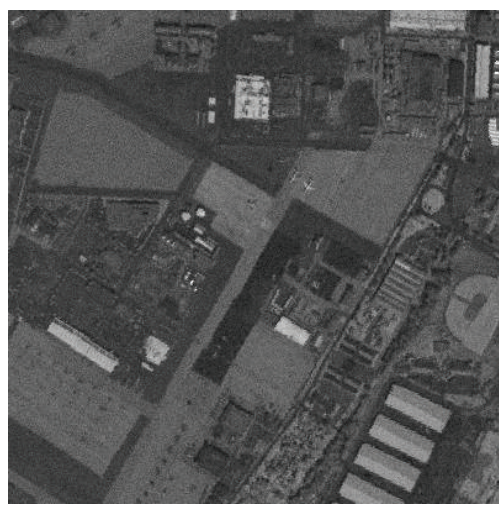

(a) Noisy HSI

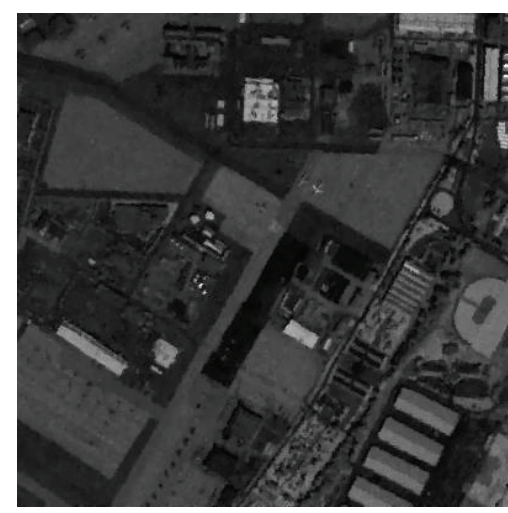

(b) CTVM

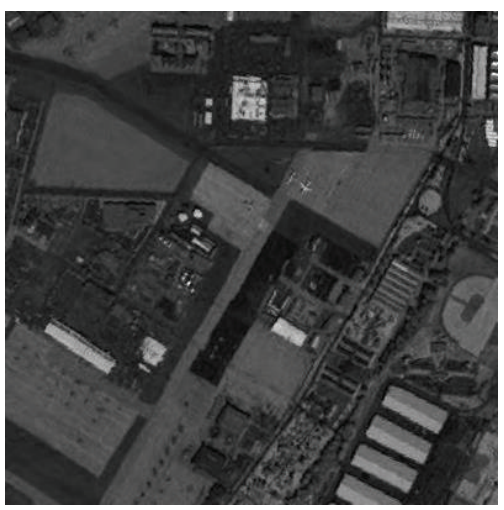

(c) TwoS

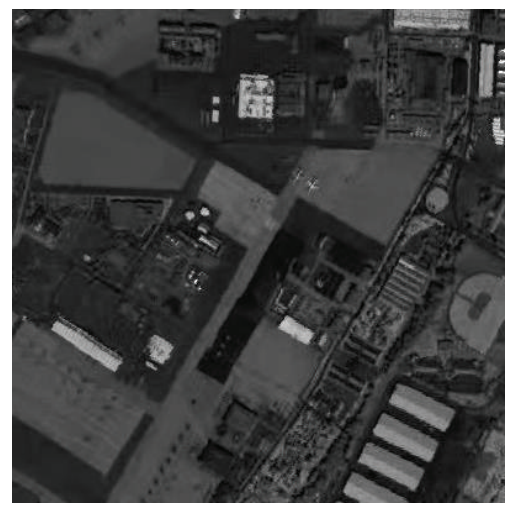

(d) PCA+WS

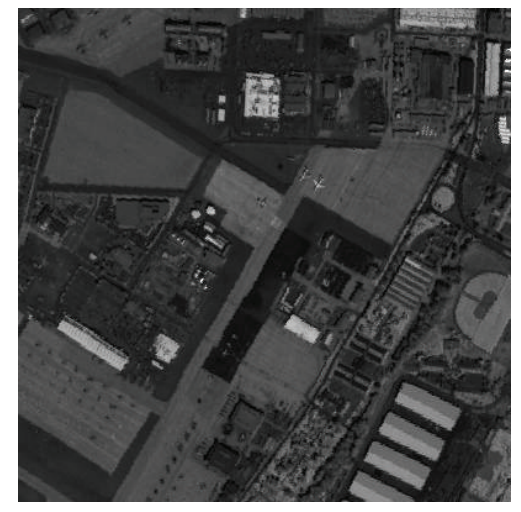

(e) Our method

FIgURE 3: Denoising results with $\sigma=15$. 


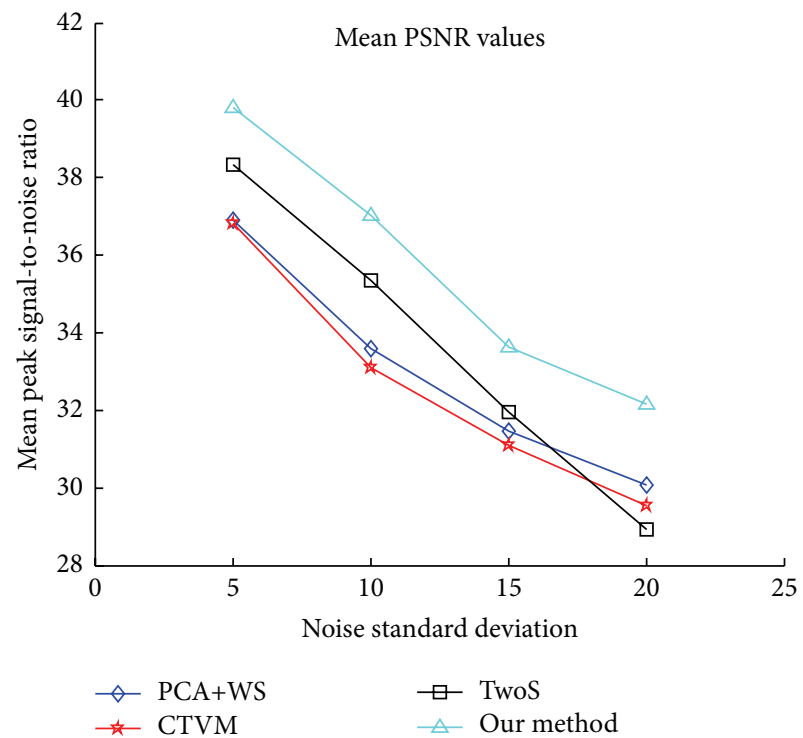

(a) Mean PSNR curves

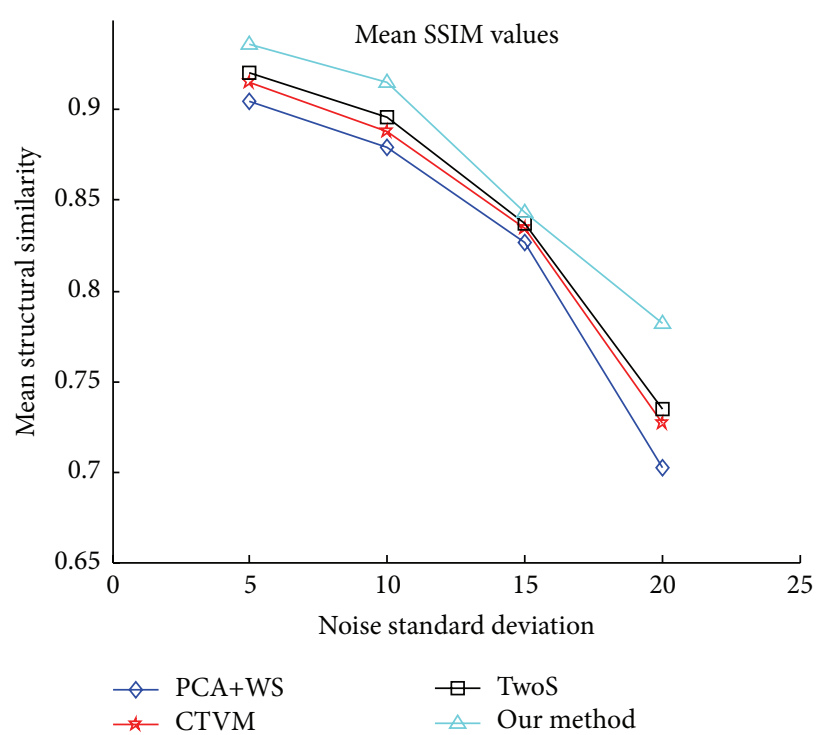

(b) Mean SSIM curves

FIGURE 4: The objective indicator for HSI denoising.

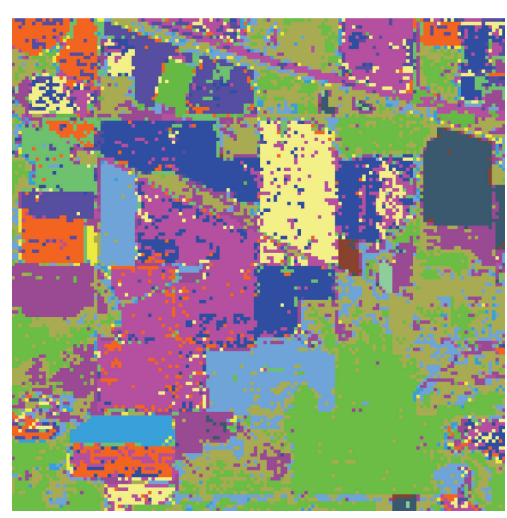

(a) Classification of noisy HSI

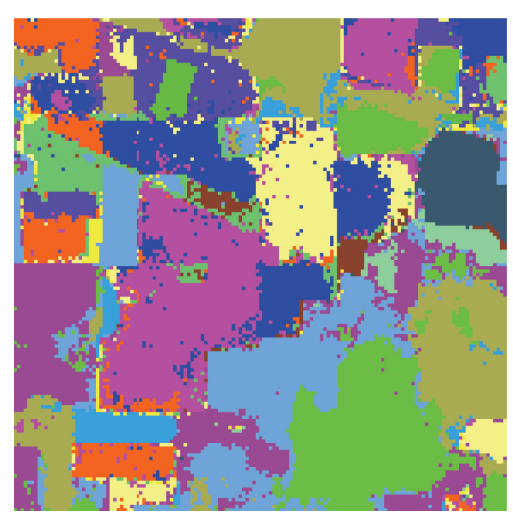

(b) Classification result of PCA+WS

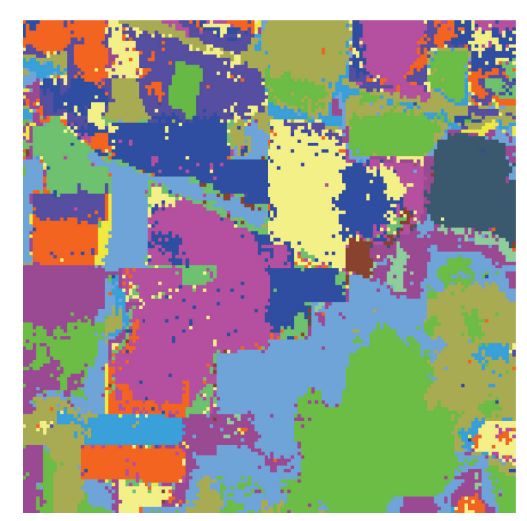

(c) Classification of CTVM

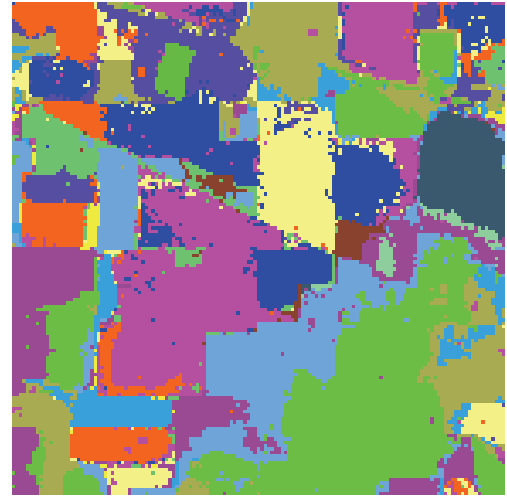

(d) Classification of TwoS

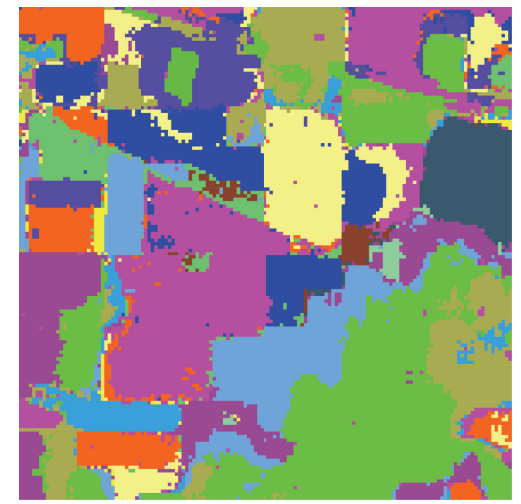

(e) Classification of our method

FIGURE 5: Classification results with several methods. 
illustrates that our method has the excellent spatial-spectral activity ability and produced less spectral distortion, which in belief is a significant factor to classification performance.

\section{Conclusion}

In this paper, a novel algorithm for HSI denoising with composite regularization models was proposed. The proposed method includes two main contributions. First, in order to utilize the spatial-spectral information simultaneously, a specific method is developed to extract a patch as a basic processing unit by reshaping the HSI cube into a $2 \mathrm{D}$ correlation matrix. Second, to overcome the shortcomings of a single prior, a framework with composite priors is used to characterize local smoothness and nonlocal group sparsity based on the extracted patch, which preserves more essential details. Compared to other state-of-the-art HSI denoising methods, our proposed method achieves competitive performance in both objective assessment and visual quality.

\section{Competing Interests}

The authors declare that there are no competing interests regarding the publication of this paper.

\section{Acknowledgments}

The paper is supported in part by National Natural Science Foundation of China (Grant 61501147), Natural Science Foundation of Heilongjiang Province (Grant F2015040), and Postdoctoral Science Foundation of Heilongjiang Province (Grant LBH-Z15099).

\section{References}

[1] C. A. Bishop, J. G. Liu, and P. J. Mason, "Hyperspectral remote sensing for mineral exploration in Pulang, Yunnan province, China," International Journal of Remote Sensing, vol. 32, no. 9, pp. 2409-2426, 2011.

[2] A. Plaza, J. M. Bioucas-Dias, A. Simic, and W. J. Blackwell, "Foreword to the special issue on hyperspectral image and signal processing," IEEE Journal of Selected Topics in Applied Earth Observations and Remote Sensing, vol. 5, no. 2, pp. 347-353, 2012.

[3] A. F. H. Goetz, "Three decades of hyperspectral remote sensing of the Earth: a personal view," Remote Sensing of Environment, vol. 113, no. S1, pp. S5-S16, 2009.

[4] A. Plaza, J. A. Benediktsson, J. W. Boardman et al., "Recent advances in techniques for hyperspectral image processing," Remote Sensing of Environment, vol. 113, supplement 1, pp. S110S122, 2009.

[5] L. Zhang and X. Huang, "Object-oriented subspace analysis for airborne hyperspectral remote sensing imagery," Neurocomputing, vol. 73, no. 4-6, pp. 927-936, 2010.

[6] M. B. Lopes, J.-C. Wolff, J. M. Bioucas-Dias, and M. A. T. Figueiredo, "Near-infrared hyperspectral unmixing based on a minimum volume criterion for fast and accurate chemometric characterization of counterfeit tablets," Analytical Chemistry, vol. 82, no. 4, pp. 1462-1469, 2010.

[7] H. Othman and S.-E. Qian, "Noise reduction of hyperspectral imagery using hybrid spatial-spectral derivative-domain wavelet shrinkage," IEEE Transactions on Geoscience and Remote Sensing, vol. 44, no. 2, pp. 397-408, 2006.

[8] G. Chen and S.-E. Qian, "Denoising of hyperspectral imagery using principal component analysis and wavelet shrinkage," IEEE Transactions on Geoscience and Remote Sensing, vol. 49, no. 3, pp. 973-980, 2011.

[9] A. Beck and M. Teboulle, "Fast gradient-based algorithms for constrained total variation image denoising and deblurring problems," IEEE Transactions on Image Processing, vol. 18, no. 11, pp. 2419-2434, 2009.

[10] Y. Huang, M. K. Ng, and Y.-W. Wen, "A fast total variation minimization method for image restoration," Multiscale Modeling \& Simulation, vol. 7, no. 2, pp. 774-795, 2008.

[11] C. Li, W. Yin, H. Jiang, and Y. Zhang, "An efficient augmented Lagrangian method with applications to total variation minimization," Computational Optimization and Applications, vol. 56, no. 3, pp. 507-530, 2013.

[12] H. Zhang, "Hyperspectral image denoising with cubic total variation model," International Society of Photogrammetry and Remote Sensing Congress (ISPRS2012), vol. I-7, pp. 95-98, 2012.

[13] Q. Yuan, L. Zhang, and H. Shen, "Hyperspectral image denoising employing a spectral-spatial adaptive total variation model," IEEE Transactions on Geoscience and Remote Sensing, vol. 50, no. 10, pp. 3660-3677, 2012.

[14] W. Liao, J. Aelterman, H. Q. Luong, A. Pižurica, and W. Philips, "Two-stage denoising method for hyperspectral images combining KPCA and total variation," in Proceedings of the 20th IEEE International Conference on Image Processing (ICIP '13), pp. 2048-2052, September 2013.

[15] J. Zhang, D. Zhao, R. Xiong, S. Ma, and W. Gao, "Image restoration using joint statistical modeling in a space-Transform domain," IEEE Transactions on Circuits and Systems for Video Technology, vol. 24, no. 6, pp. 915-928, 2014.

[16] A. Li and H. Shouno, "Dictionary-based image denoising by fused-lasso atom selection," Mathematical Problems in Engineering, vol. 2014, Article ID 368602, 10 pages, 2014.

[17] J. Mairal, F. Bach, J. Ponce, G. Sapiro, and A. Zisserman, "Nonlocal sparse models for image restoration," in Proceedings of the 12th International Conference on Computer Vision (ICCV '09), pp. 2272-2279, Kyoto, Japan, October 2009.

[18] K. Dabov, A. Foi, V. Katkovnik, and K. Egiazarian, "Image denoising by sparse 3-D transform-domain collaborative filtering," IEEE Transactions on Image Processing, vol. 16, no. 8, pp. 2080-2095, 2007.

[19] B. Rasti, J. R. Sveinsson, M. O. Ulfarsson, and J. A. Benediktsson, "Hyperspectral image denoising using a new linear model and Sparse Regularization," in Proceedings of the 33rd IEEE International Geoscience and Remote Sensing Symposium (IGARSS '13), pp. 457-460, Melbourne, Australia, July 2013.

[20] F. Palsson, M. O. Ulfarsson, and J. R. Sveinsson, "Hyperspectral image denoising using a sparse low rank model and dual-tree complex wavelet transform," in Proceedings of the IEEE International Geoscience and Remote Sensing Symposium (IGARSS '14), pp. 3670-3673, Quebec City, Canada, July 2014.

[21] Y.-Q. Zhao and J. Yang, "Hyperspectral image denoising via sparse representation and low-rank constraint," IEEE Transactions on Geoscience and Remote Sensing, vol. 53, no. 1, pp. 296308, 2015.

[22] H. Chang, X. Zhang, X.-C. Tai, and D. Yang, "Domain decomposition methods for nonlocal total variation image restoration," Journal of Scientific Computing, vol. 60, no. 1, pp. 79-100, 2014. 
[23] M. Fornasier, Y. Kim, A. Langer, and C.-B. Schönlieb, "Wavelet decomposition method for $L_{2} / \mathrm{TV}$-image deblurring," SIAM Journal on Imaging Sciences, vol. 5, no. 3, pp. 857-885, 2012.

[24] S. S. Chen, D. L. Donoho, and M. A. Saunders, "Atomic decomposition by basis pursuit," SIAM Review, vol. 43, no. 1, pp. 129$159,2001$.

[25] J. A. Tropp and A. C. Gilbert, "Signal recovery from random measurements via orthogonal matching pursuit," IEEE Transactions on Information Theory, vol. 53, no. 12, pp. 4655-4666, 2007.

[26] J. M. Bioucas-Dias and M. A. Figueiredo, "A new TwIST: two-step iterative shrinkage/thresholding algorithms for image restoration," IEEE Transactions on Image Processing, vol. 16, no. 12, pp. 2992-3004, 2007.

[27] T. Goldstein and S. Osher, "The split Bregman algorithm for L1 regularized problems," SIAM Journal on Imaging Sciences, vol. 2, no. 2, pp. 323-343, 2009.

[28] A. Buades, B. Coll, and J.-M. Morel, "A non-local algorithm for image denoising," in Proceedings of the IEEE Computer Society Conference on Computer Vision and Pattern Recognition (CVPR '05), vol. 2, pp. 60-65, June 2005.

[29] J. V. Manjón, P. Coupé, A. Buades, D. L. Collins, and M. Robles, "New methods for MRI denoising based on sparseness and selfsimilarity," Medical Image Analysis, vol. 16, no. 1, pp. 18-27, 2012.

[30] L. Ao, L. Yibing, Y. Xiaodong, and L. Yue, "Image restoration with dual-prior constraint models based on Split Bregman," Optical Review, vol. 20, no. 6, pp. 491-495, 2013.

[31] J. Liu, H. Ding, and S. Molloi, "Nonlocal total variation based spectral CT image reconstruction," Medical Physics, vol. 42, no. 6, p. 3570, 2015.

[32] W. Dong, L. Zhang, G. Shi, and X. Li, "Nonlocally centralized sparse representation for image restoration," IEEE Transactions on Image Processing, vol. 22, no. 4, pp. 1620-1630, 2013.

[33] S. Boyd, N. Parikh, E. Chu, B. Peleato, and J. Eckstein, "Distributed optimization and statistical learning via the alternating direction method of multipliers," Foundations and Trends in Machine Learning, vol. 3, no. 1, pp. 1-122, 2010.

[34] A. Beck and M. Teboulle, "A fast iterative shrinkage-thresholding algorithm for linear inverse problems," SIAM Journal on Imaging Sciences, vol. 2, no. 1, pp. 183-202, 2009.

[35] J. Zhang, Z.-H. Wei, and L. Xiao, "A relaxed split bregman iteration for total variation regularized image denoising," Intelligent Computing Theories and Applications, vol. 7390, pp. 189-197, 2012.

[36] A. Rehman and Z. Wang, "Reduced-reference image quality assessment by structural similarity estimation," IEEE Transactions on Image Processing, vol. 21, no. 8, pp. 3378-3389, 2012. 


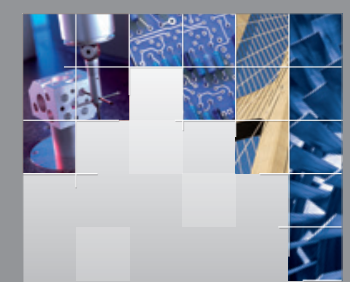

\section{Enfincering}
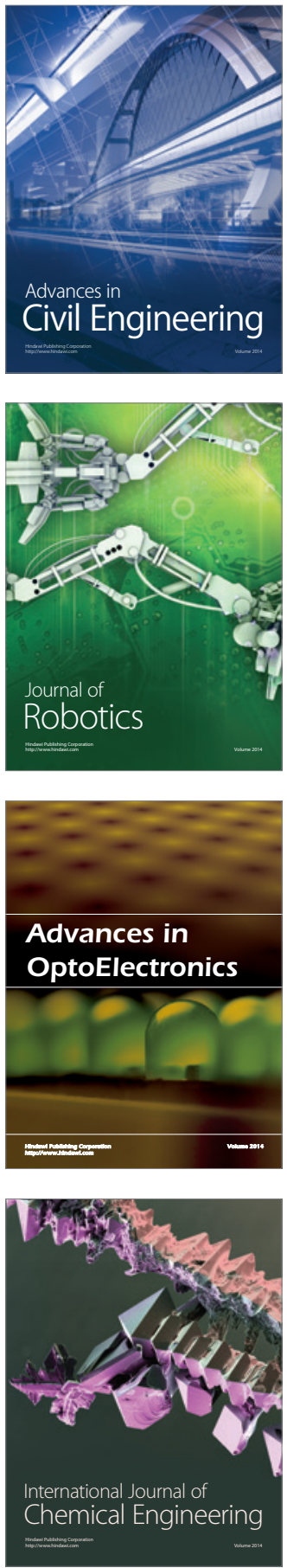

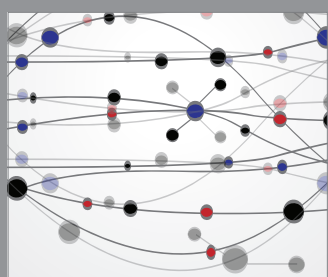

The Scientific World Journal

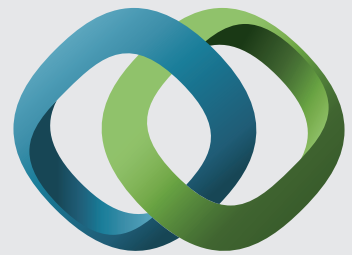

\section{Hindawi}

Submit your manuscripts at

http://www.hindawi.com
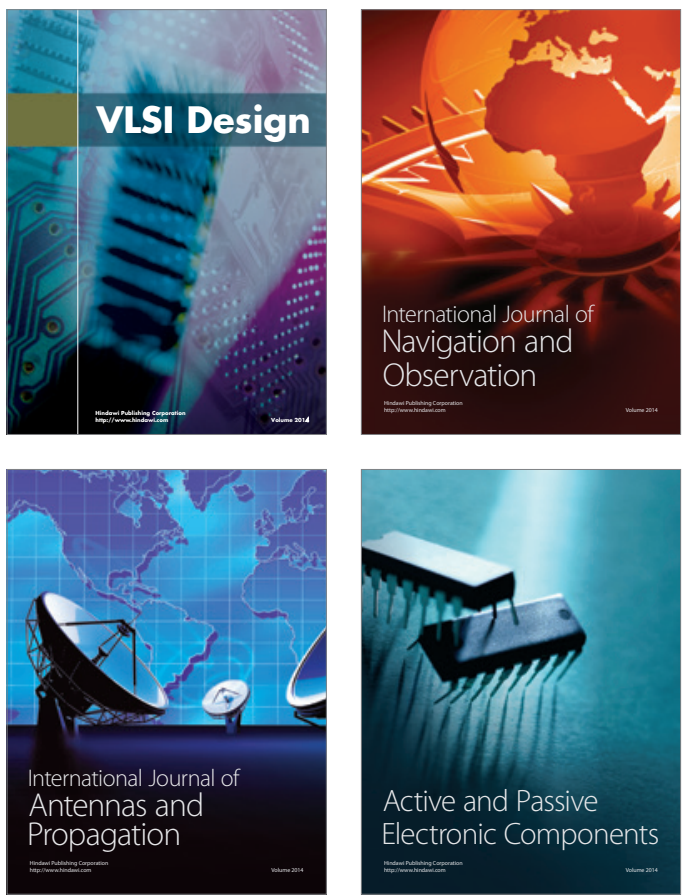
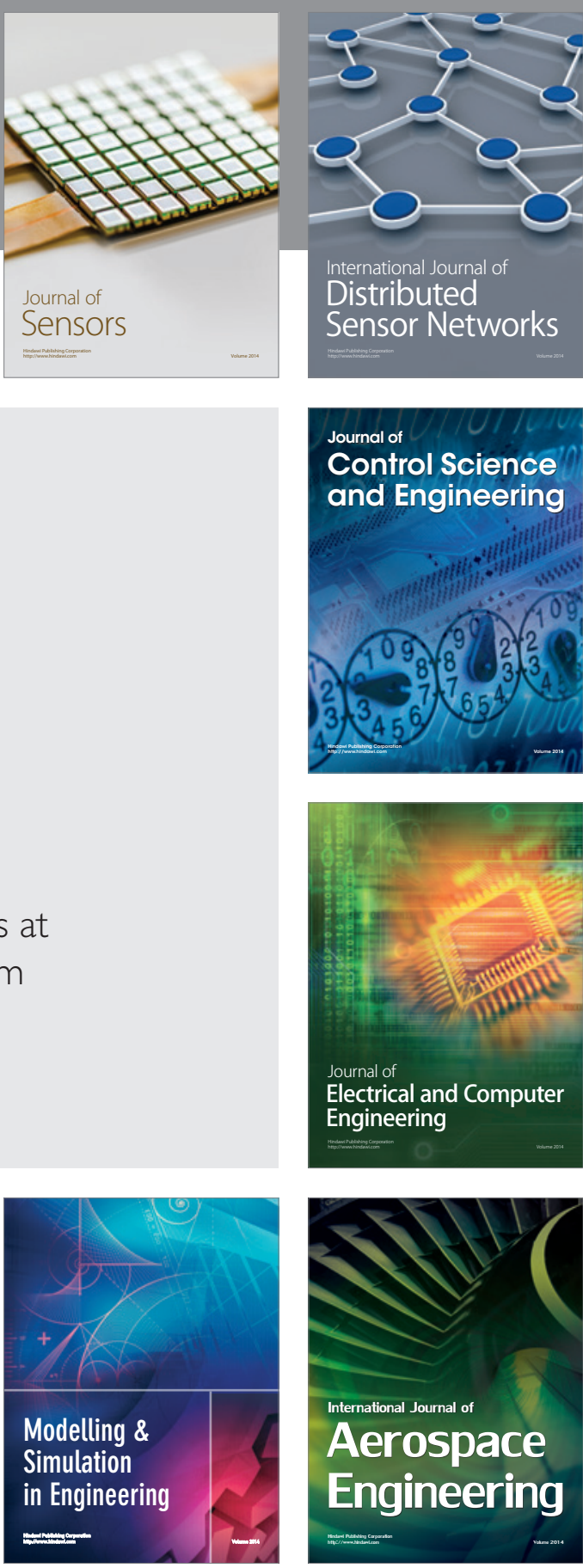

International Journal of

Distributed

Sensor Networks

Journal of

Control Science

and Engineering
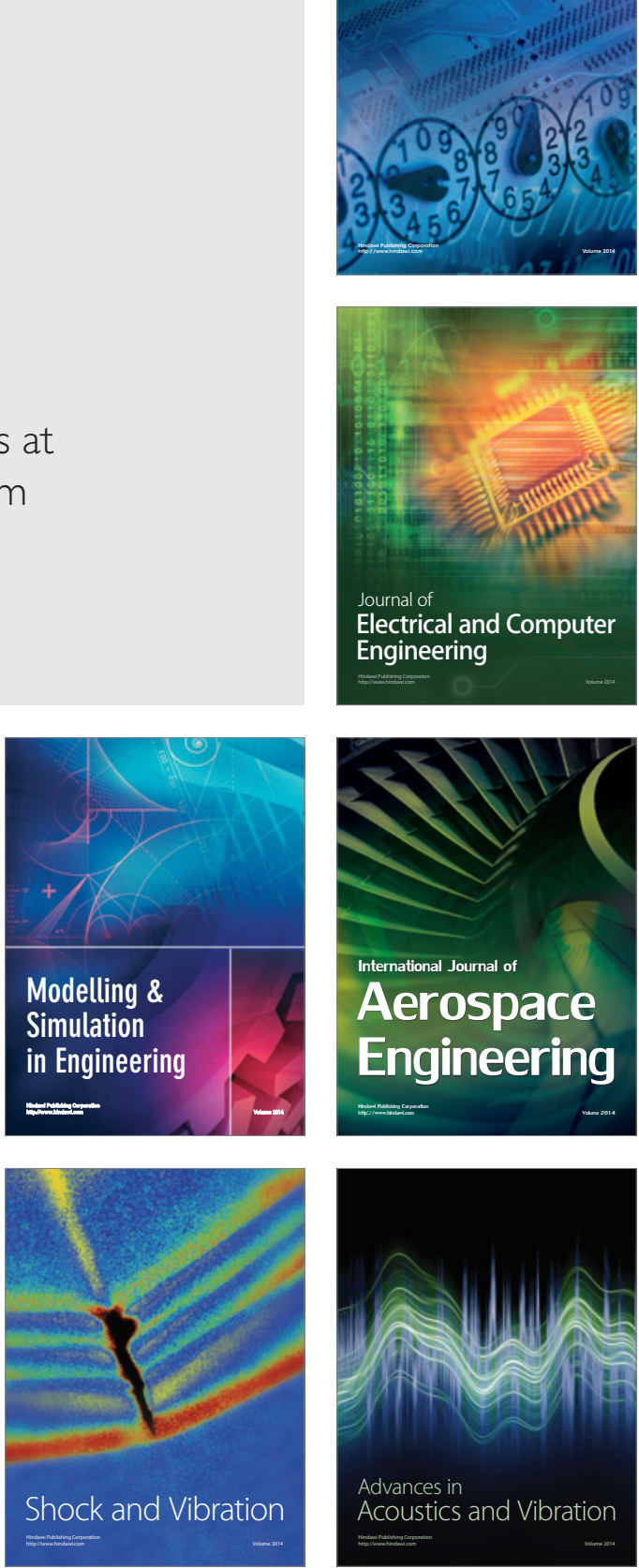